\section{The impact of medical clowns exposure over postoperative pain and anxiety in children and caregivers: An Israeli experience}

\author{
Nitza Newman,1 Slava Kogan,2,3 \\ Moshe Stavsky, ${ }^{2,3}$ Shay Pintov, ${ }^{4}$
}

Yotam Lior ${ }^{2,3}$

1Pediatric Surgery Department, Faculty of Health Sciences, Soroka University Medical Center, Ben-Gurion University of the Negev, Beer-Sheva; ${ }^{2}$ Joyce and Irwing Goldman Medical School, Faculty of Health Sciences, Ben-Gurion University of the Negev, Beer-Sheva; 3Soroka Clinical Research Center, Soroka University Medical Center, BeerSheva; ${ }^{4}$ The Medical School for International Health, Faculty of Health Sciences, Ben Gurion University of the Negev, Beer-Sheva, Israel

\begin{abstract}
While postoperative pain management was shown to reduce unwanted physiological and emotional outcomes, pediatric postoperative pain management remains suboptimal. Medical-clowns were shown to be beneficial in many medical contexts including reduction of stress, anxiety and pain. This study was set to assess the effectiveness of medical-clowns on pediatric postoperative pain reduction. Children age 4 or above, planned for elective hernia repair surgery were recruited. Children were randomly divided to a control or medicalclown escorted groups. Demographical and clinical data were collected using questionnaires and electronic sheets. Children escorted by clowns reported lower levels of pain upon admittance, discharge and 12hours post-surgery. Statistically significant reduction of parental distress and significantly higher serum cortisol levels were observed in the clown-therapy group. Although small, our study supports the possibility that preoperative medical-clown therapy might be a cheap, safe and yet beneficial method for postoperative pain reduction.
\end{abstract}

\section{Introduction}

The current definition of pain according to the International Association for the Study of Pain (AISP) is "an unpleasant sensory and emotional experience associated with actual or potential tissue damage or described in terms of such damage". ${ }^{1}$ While most medical interventions are conducted with intent to improve the patient's quality of life while minimizing pain and discomfort, some invasive interventions such as surgery necessitate some degree of collateral tissue damage and thus are expected to result in post-operative pain. Left untreated, pain has been shown to result in multiple adverse physiological and emotional outcomes including immobility, ${ }^{2}$ atrophy, ${ }^{3}$ sleep disturbance, 4,5 anxiety and depression. ${ }^{6}$ Therefore, it comes to no surprise that effective management of postoperative pain is of outmost concern for both patient and surgeon.

While traditional post-operative pain management is mainly conducted pharmaceutically using opioids, NSAIDs and acetaminophen, more modern approaches call for a multimodal approach addressing both the patient's mental condition such as mental stress in addition to known physical conditions as both were shown to affect postoperative pain. 7,8

Alongside great advances in awareness, approach and means for optimal postoperative pain management in adults, pediatric postoperative pain management is still considered to be suboptimal in many medical centers. ${ }^{9}$ Some of the identified leading factors to inadequate pain management among children are difficulties in pain assessment and quantification, age restricted drug prescriptions, ${ }^{9}$ inter-patient variability in pain perception and analgesic requirements, ${ }^{10}$ pharmacodynamics and pharmacokinetics variance in age and ethnicity and scarcity of studies examining the effects of analgesics among children. ${ }^{11,12}$ The will to improve postoperative pain management in children served as a catalysator to growing interest in nonpharmacological techniques, mostly relying on perioperative stress and anxiety reduction by various means. ${ }^{9}$

To the majority of population, the word 'clown' refers to a comic performer, usually in extravagant costumes, whose main aim is to entertain crowds of various ages. While similar in behavior and often costume, medical clowns are specifically trained to attend the psycho-social needs of hospitalized children, adults and even the elderly.13-16 According to a child's age, language, ability and intellect, the medical clown individually applies music, jokes, improvisations, mimicry and juggling to best form a connection. This connection is then used to alleviate stress and anxiety often related to the unfamiliar hospital environment, physi-
Correspondence: Yotam Lior, Soroka Clinical Reseach Center, Soroka University Medical Center, P.O.B 151, Beer-Sheva, 84101, Israel.

Tel: +972-549987470

E-mail: yotam.lior1@gmail.com

Key words: pain reduction, hernia, clown-doctors, cortisol, pediatric surgery.

Contributions: NW: data collection, manuscript reviewing, SK: data collection, MS: data collection, SP: manuscript reviewing, YL: data collection, data analysis, manuscript writing, manuscript reviewing.

Conflict of interests: the authors declare no potential conflict of interests.

Funding: the work was supported by "The Dream Doctors Project" non-profit organization, Israel.

Received for publication: 2 May 2019

Revision received: 4 June 2019.

Accepted for publication: 5 June 2019.

This work is licensed under a Creative Commons Attribution NonCommercial 4.0 License (CC BY-NC 4.0)

(C) Copyright: the Author(s), 2019

Licensee PAGEPress, Italy

Pediatric Reports 2019; 11:8165

doi:10.4081/pr.2019.8165

cal pain and emotional distress. ${ }^{17}$

Since the introduction and acceptance of the medical clowning profession to the medical community in the early nineties, a large amount of literature has been published regarding beneficial effects of humor-therapy in clinical context. Humor was found to have beneficial effect on the immune system, ${ }^{18-21}$ as well as promote a faster resolution of respiratory symptoms in children. ${ }^{22}$ Humor and medical clowning were found to alleviate chronic and acute pain associated with medical conditions and procedures, ${ }^{23-27}$ improve mental health status and functions 28,29 and were even associated with improved IVF outcomes. ${ }^{14}$

Medicinal treatments, especially medical operations, are well-known stressors for both children and adults. However, while the adult patient is usually capable of communicating her or his concerns, understand the necessity of an operation and comply with physician requests, a child is often unable to do so due to language and understanding limitations. Furthermore, many children are brought to the unfamiliar environment of the medical facility unaware of upcoming operation, which further increases situation-associated stress. 
Due to limited communication and analysis abilities, young and very-young children often react to situations by interpreting their parents body language and expressions. ${ }^{30}$ Given that many parents are anxious before an operation of a loved one, this natural behavior serves to further increase the preoperative anxiety of a child.

The ability of the medical clown to interact with young children as well as their parents prior to an operation was studied in the past and found to be effective in the reduction of preoperative anxiety, stress and worries in both children and parents. $31-34$ However, most of these studies focused on the preoperative aspect only and utilized a questionnaire-only approach in the assessment of medical clown therapy effectiveness.

The main objective of this study was to assess the efficacy of preoperational medical clown therapy on post-operative pain and stress utilizing both standard questionnaires as well as serum cortisol as an objective stress marker.

This study was approved by the Soroka University Medical Center (SUMC) and the Assuta Medical Center (AMC) ethics committees. The study is registered in the ClinicalTrials.gov register, identifier no. NCT01622218

\section{Materials and Methods}

\section{Study population}

Children above the age of 4 who were listed for an elective umbilical or inguinal hernia repair surgery under general anesthesia in the Be'er-Sheva's Assuta Medical Center (AMC) or Soroka University Medical Center (SUMC), a major tertiary hospital in the southern part of Israel, between the years 2012-2015 were considered eligible for this study. Children with medical history of chronic illness, previous operations or mental retardation were excluded from the study to as well as children with American Society of Anesthesiologists Physical Status (ASAPS) greater than 1 or children presenting with fever prior to operation.

\section{Study procedure}

After enrolment, children were randomly assigned to one of two research arms: (A) medical clown group, in which children and parents were accompanied by a medical clown from admission to post-anesthesia care unit, or (B) control group, in which children and parents underwent the standard procedure.

In the medical clown group, preopera- tive interaction time ranged between 30 to 60 minutes. The clown interacted with both child and parents using various methods fitted to the child's age, language and mental ability (e.g. balloons, puppets, word games, magic tricks, dice tricks, jokes etc').

The medical clown then escorted the child to the operation theater and stayed there until complete anesthesia was confirmed. After surgery completion, the medical clown waited for the child in the postoperative care unit and soothingly interacted with him or her upon emergence.

All operations were performed between 15:30 and 19:30 by board-certified pediatric surgeons applying the open technique. In all cases anesthesia was administered by board-certified pediatric-anesthesia-experienced anesthesiologist utilizing a scented mask and standard oxygen / nitrous oxide / sevoflurane mix technique.

\section{Data collection and evaluation instruments}

Once informed consent was obtained, demographical and physiological data was collected from child and parents as well as from medical records. This was followed by pre-operative pain assessment of the child using the Wong Baker Faces Pain Rating Scale (WBS) and pre-operative distress assessment of the child's parents using Subjective Units of Distress (SUD) scale scores. Parental post-operative SUD was assessed immediately after operation. The child's post-operative WBS score was obtain immediately after surgery and parents were asked to record and report WBS scores at 3,12- and 24-hours post-operation. To standardize post-operative analgesic therapy, parents were dispensed 24 hours aliquots of paracetamol syrup (25 $\mathrm{mg} / \mathrm{mL}$, ACAMOLI, Teva Pharmaceutical Industries, Israel) according to the child's body weight. Parents were then asked to quantitively record and report the amount of pain-relief used in the first 24 hours after surgery.

\section{Wong-Baker Faces Pain Rating Scale}

The Wong-Baker Faces Pain Rating Scale was developed by Donna Wong and Connie Baker for the assessment of pain in children. Briefly, the child is presented with a series of faces ranging from happy face to a crying face, which are allocated to 0 or "no pain" and 10 or "hurts worst", respectively. The child is then encouraged to pick the face which best describes his or her current feeling. Given that the child does not need to verbally describe the physical feeling for the primary care giver to assess the child's pain, the WBS is a valuable tool for pain assessment in very young or shy children. The scale was found to be a reliable and effective mean for pain assessment in children in several studies. ${ }^{35-40}$

\section{Subjective Units of Distress Scale}

Also known as Subjective Units of Disturbance scale, the SUD scale was developed by Joseph Wolpe in 1969 as a mean for subjective personal distress assessment. ${ }^{41}$ Briefly, the participant is asked to assess his or her current subjective distress on a scale of 0 to 10 with 0 being complete serenity and 10 being immense distress. The SUD score has been used as a reliable tool for the evaluation of subjective distress in several studies to date. ${ }^{42-45}$

\section{Serum Cortisol levels as a biomark- er of stress}

Serum cortisol was measures as biomarker for physiological stress. After surgical anesthesia induction and the insertion of a peripheral intravenous catheter, 5 $\mathrm{mL}$ blood samples were obtained from each child, prior to injection of drugs or saline. Samples were immediately centrifuged for serum separation and frozen until analysis. All samples were obtained between 15:30 and 19:30. Serum cortisol levels were measured using Advia Centaur XP (Siemens Healthineers, Erlangen, Germany).

\section{Statistical analysis}

Statistical analysis in this study was performed using IBM SPSS. The method of analyses for continuous variables was parametric using student t-test. Non-parametric procedures were used if parametric assumptions could not be satisfied and included the Mann-Whitney test. Parametric model assumptions were assessed using Normalplot or Shapiro-Wilks statistic for verification of normality and Levene's test for verification of homogeneity of variances. Categorical variables were tested using Pearson's $\chi^{2}$ test for contingency tables or Fisher Exact test, as appropriate.

All statistical tests and/or confidence intervals, as appropriate, were performed at $\alpha=0.05$ (2-sided). All P-values reported will be rounded to two decimal places.

\section{Results}

A total of 45 children were enrolled to the study. Cohort characteristics are described in Table 1. Although small variances exist, both groups were comparable in all assessed variables. 
Clinical outcomes are detailed in Table 2. No statistically significant difference was observed between the two groups in terms of WBS scores. However, lower WBS scores were recorded among children in the clown group upon admittance $(0.31 \pm 0.47$ vs. $0.5 \pm 1.79)$, discharge $(3.91 \pm 3.79 v s$. $4.41 \pm 3.12, \mathrm{P}=0.32)$ and the 12-hour interval ( $2.77 \pm 2.86 v s .3 .47 \pm 3.31, \mathrm{P}=0.32)$. The two groups were comparable in terms of 24 hours post-surgical analgesic care.

While parents in the clown group reported higher levels of admission SUD (5.48 $\pm 3.06 v s$. $3.48 \pm 3.31, \mathrm{P}=0.06)$, both groups reported similar SUD levels at discharge, thus marking a statistically significant reduction in SUD score in the clown group $(-2.97 \pm 2.75 v s .-0.81 \pm 2.51, \mathrm{P}=0.02)$.

Intraoperative serum cortisol levels of children in the clown group were significantly higher than those of the control group (12.52 \pm 6.14 vs. $7.46 \pm 5.6, \mathrm{P}=0.0004)$.

\section{Discussion}

It is estimated the as many as $60 \%$ of children will experience significant anxiety prior to operation, ${ }^{46}$ an anxiety estimated to

Table 1. Cohort characteristics.

\begin{tabular}{|c|c|c|c|}
\hline Variable & $\begin{array}{l}\text { Control Group } \\
(\mathrm{n}=22)\end{array}$ & $\begin{array}{c}\text { Clown Group } \\
(n=23)\end{array}$ & P-value \\
\hline Age, mean (SD) & $5.67 \pm 1.47$ & $5.91 \pm 1.46$ & 0.51 \\
\hline Sex, male, № (\%) & $14(63.6)$ & $12(52.2)$ & 0.44 \\
\hline $\begin{array}{l}\text { Ethnicity, No (\%) } \\
\text { Non-Jewish } \\
\text { Jewish }\end{array}$ & $\begin{array}{l}11(50) \\
11(50)\end{array}$ & $\begin{array}{c}6(26.1) \\
17(73.9)\end{array}$ & 0.1 \\
\hline $\begin{array}{l}\text { Mother tongue, No (\%) } \\
\text { Hebrew } \\
\text { Arabic }\end{array}$ & $\begin{array}{l}10(47.6) \\
11(52.4)\end{array}$ & $\begin{array}{c}16(72.7) \\
6(27.3)\end{array}$ & 0.09 \\
\hline Weight (Kg), mean (SD) & $20.75(5.67)$ & $20.82(3.27)$ & 0.58 \\
\hline Temperature $\left(\mathrm{C}^{\circ}\right)$, mean $(\mathrm{SD})$ & $36.41(0.38)$ & $36.54(0.4)$ & 0.3 \\
\hline Father's education (years), mean (SD) & $11.9(1.41)$ & $12.87(3.17)$ & 0.07 \\
\hline Mother's education (years), mean (SD) & $12.47(2.86)$ & $13.57(3.3)$ & 0.08 \\
\hline
\end{tabular}

Table 2. Clinical outcomes.

\begin{tabular}{lccc} 
Variable & $\begin{array}{c}\text { Control Group } \\
(\mathrm{n}=22)\end{array}$ & $\begin{array}{c}\text { Clown Group } \\
(\mathrm{n}=23)\end{array}$ & $\begin{array}{c}\text { P-value } \\
\text { WBS, mean (SD) }\end{array}$ \\
$\quad$ Admittance & $0.5(1.79)$ & $0.31(0.47)$ & 0.63 \\
Discharge & $4.41(3.12)$ & $3.91(3.79)$ & 0.32 \\
12 hours & $3.47(3.31)$ & $2.77(2.86)$ & 0.31 \\
24 hours & $2.56(2.36)$ & $2.55(2.7)$ & 0.91 \\
Analgesic care, mean (SD) & $0.73(0.77)$ & $1.13(1.29)$ & 0.44 \\
\hline Parental SUD, mean (SD) & & & \\
$\quad$ Admittance & $3.48(3.31)$ & $5.48(3.06)$ & 0.06 \\
$\quad$ Discharge & $2.44(2.63)$ & $2.43(2.51)$ & 0.95 \\
$\quad$ Delta & $-0.81(2.51)$ & $-2.97(2.75)$ & 0.02 \\
Serum cortisol (mcg/dL), mean (SD) & $7.46(5.6)$ & $12.52(6.14)$ & 0.004 \\
\hline
\end{tabular}

be significantly affected by that of an escorting parent. 47 Anxiety has been shown to exacerbate experienced pain and has thus been the target of novel approaches for pain reduction, especially in population where pharmaceutical intervention is considered more complicated.48 Among the various methods attempted for preoperative anxiety reduction are video directions, 49,50 patient education and orientation, 51,52 sedation, 53 music, 54 games, 55 and medicinal clowns intervention. ${ }^{13,32,56,57}$ While all methods were shown to be effective in terms of preoperative anxiety reduction, only a few were assessed for perceived postoperative pain, even fewer were assessed for 24-hour effects.

Several studies have shown that medical clowns exposure reduces preoperative anxiety among children.24,25,31,32,56 However, very few assess the impact of medical clown therapy over parental stress, in spite having the connection between parental anxiety and behavioral and emotional responses in children previously described. ${ }^{31,58}$ In our study we have shown that parents exposed to medical clowning have experienced a significantly bigger stress reduction compared with nonexposed parents (delta from admittance -
$2.97 \pm 2.75$ vs. $-0.81 \pm 2.51$, respectively, $\mathrm{P}=0.02$ ), similar to the results of Vagnoli et al. 31 This stress reduction could be perceived by the child and facilitate the reduction of infant anxiety and associated pain, however more research needs to be done to support this theory. It is important to note however, that final SUD levels of both groups were found to be similar $(2.44 \pm 2.64$ vs. $2.43 \pm 2.51$ for exposed and non-exposed parents, respectively) and that the observed delta effect could possibly be related to initial parental SUD levels rather than the intervention itself.

In our study we have found that children exposed to medical clowns repeatedly reported lower WBS scores, albeit without statistical significance, probably due to small sample size. These results are in agreement with the result shown by Kovherove et al. who have shown that medical clowns treatment resulted in a significant reduction in perceived preoperative as well as postoperative pain in operated children. 23

While expecting a reduction in serum cortisol levels among children in the medical clown group, we were surprised to find significantly higher levels $(12.52 \pm 6.14 \mathrm{vs}$. $7.46 \pm 5.6, \mathrm{P}=0.004)$. It would seem that the effect of medical clowns on cortisol levels is incoherent and may lead to an increase like the one found in our study, a decrease like in the one found in the work of Sanchez et at. ${ }^{59}$ or have no effect like in the work of Rimon et al. 60 While surprising, the statistical significance of this finding raises the question whether cortisol levels may reflect the child interest and psycho-cognitive engagement with the clown. However, further research is needed to answer this question.

Our results demonstrate a possible connection between parental emotional response and pediatric emotional and physical experience. However, one of the challenges our and similar studies have faced is the lack of relevant information regarding parental personality traits, emotional coping mechanisms, resilience and mental status, all of which might alter their response to the situation. While this information could have indeed potentially improved our insights, one has to consider the challenge, difficulty and potential biases of asking a parent escorting his child to a stressful medical procedure to cooperate with personality tests. However, we do hope that future studies will be able to include these important aspects in their assessments.

In order to minimize confounding effects in our study we have chosen to use randomization when assigning children to each of the groups. However, it is not com- 
pletely impossible that minor uncontrolled alterations might still exist. Alterations which might affect the overall perceived outcome and be overemphasized in a relatively small cohort such as our own. Such alterations, not recoded or controlled for, might include presurgical anticipation of events, prolong motor agitation upon anesthesia emergence, socio-economical background, past parental experiences or even surgeon and anesthesiologist approach. However, we still believe that the best method the minimize such possible effects is randomization but acknowledge that larger cohort could have further strengthened our study. Furthermore, we believe that a larger cohort could have possibly even shift some of the trends depicted in this study toward statistical significance. We therefore hope that future studies will be composed of larger cohorts in order to achieve this goal. Other weakness such as variances between surgeons and surgical theaters may exists but are assumed to have minimal effect over the results of the study.

\section{Conclusions}

While many physicians and medical staff consider medical clowns to be a distraction or even a nuisance in surgical theater routine, our study shows that medical clown interactions may be a cheap, safe and effective measure for the reduction of operation-associated parental stress and maybe even postoperative pain among children. These results still need to be validated in further studies.

\section{References}

1. Bonica JJ. The need of a taxonomy. Pain 1979;6:247-8.

2. Cousins MJ, Bridenbaugh PO. Neural blockade in clinical anesthesia and management of pain: Lippincott Williams \& Wilkins; 1998.

3. Hides J, Stokes M, Saide M, et al. Evidence of lumbar multifidus muscle wasting ipsilateral to symptoms in patients with acute/subacute low back pain. Spine 1994;19:165-72.

4. Smith MT, Haythornthwaite JA. How do sleep disturbance and chronic pain inter-relate? Insights from the longitudinal and cognitive-behavioral clinical trials literature. Sleep Med Rev 2004;8: 119-32.

5. Marin R, Cyhan T, Miklos W. Sleep disturbance in patients with chronic low back pain. Am J Phys Med Rehabil
2006;85:430-5.

6. Gureje O, Simon GE, Von Korff M. A cross-national study of the course of persistent pain in primary care. Pain 2001;92:195-200.

7. Macintyre PE, Scott DA, Schug SA, et al. Acute pain management: scientific evidence. Australian and New Zealand College of Anaesthetists; 2010. Available from: https://www.mascc.org/ assets/documents/pain_Acute_Pain_A U_NZcollage_faculty.pdf

8. Lovich-Sapola J, Smith CE, Brandt CP. Postoperative pain control. The Surgical clinics of North America. 2015;95:30118.

9. Brasher C, Gafsous B, Dugue S, et al. Postoperative pain management in children and infants: an update. Paediatr Drugs 2014;16:129-40.

10. Mohammed BS, Engelhardt T, Cameron GA, et al. Population pharmacokinetics of single-dose intravenous paracetamol in children. Br J Anaesth 2012;108:823-9.

11. Kart T, Christrup LL, Rasmussen M. Recommended use of morphine in neonates, infants and children based on a literature review: Part 1Pharmacokinetics. Paediatr Anaesth 1997; 7:5-11.

12. Kart T, Christrup LL, Rasmussen M. Recommended use of morphine in neonates, infants and children based on a literature review: Part 2-Clinical use. Paediatr Anaesth 1997;7:93-101.

13. Finlay F, Baverstock A, Lenton S. Therapeutic clowning in paediatric practice. Clin Child Psychol Psychiatry 2014;19:596-605.

14. Friedler S, Glasser S, Azani L, et al. The effect of medical clowning on pregnancy rates after in vitro fertilization and embryo transfer. Fertility Sterility 2011;95:2127-30.

15. Nuttman-Shwartz O, Scheyer R, Tzioni H. Medical clowning: even adults deserve a dream. Soc Work Health Care 2010;49:581-98.

16. McMahan SC. Infinite possibility: clowning with elderly people. Care Manag J 2008;9:19-24.

17. Finlay F, Baverstock A, Lenton S. Therapeutic clowning in hospital settings: more than a play-fool proposal. Arch Dis Child 2017;102:597-8.

18. Bennett MP, Lengacher C. Humor and Laughter May Influence Health IV. Humor and Immune Function. Evidence-based complementary and alternative medicine: eCAM. 2009;6:159-64.

19. Bennett MP, Zeller JM, Rosenberg L, et al. The effect of mirthful laughter on stress and natural killer cell activity. Altern Ther Health Med 2003;9:238-45.

20. Bennett MP, Lengacher C. Humor and Laughter May Influence Health: III. Laughter and Health Outcomes. Evidence-based complementary and alternative medicine: eCAM. 2008;5:37-40.

21. Carrasco FR, Schmidt G, Romero AL, et al. Immunomodulatory activity of Zingiber officinale Roscoe, Salvia officinalis L. and Syzygium aromaticum L. essential oils: evidence for humor- and cell-mediated responses. J Pharmacy Pharmacol 2009;61:961-7.

22. Bertini M, Isola E, Paolone G, et al. Clowns benefit children hospitalized for respiratory pathologies. Evidence-based complementary and alternative medicine: eCAM. 2011;2011:879125.

23. Kocherov S, Hen Y, Jaworowski S, et al. Medical clowns reduce pre-operative anxiety, post-operative pain and medical costs in children undergoing outpatient penile surgery: A randomised controlled trial. J Paediatr Child Health 2016;52:877-81.

24. Meiri N, Ankri A, Hamad-Saied M, et al. The effect of medical clowning on reducing pain, crying, and anxiety in children aged 2-10 years old undergoing venous blood drawing - a randomized controlled study. Eur J Pediatr 2016;175:373-9.

25. Wolyniez I, Rimon A, Scolnik D, et al. The effect of a medical clown on pain during intravenous access in the pediatric emergency department: a randomized prospective pilot study. Clin Pediatr 2013;52:1168-72.

26. Meiri N, Ankri A, Hamad-Saied M, et al. The effect of medical clowning on reducing pain, crying, and anxiety in children aged 2-10 years old undergoing venous blood drawing - a randomized controlled study. Eur J Pediatr 2016;175:373-9.

27. Tse MM, Lo AP, Cheng TL, et al. Humor therapy: relieving chronic pain and enhancing happiness for older adults. J Aging Res 2010;2010:343574.

28. Gelkopf M. The use of humor in serious mental illness: a review. Evidencebased complementary and alternative medicine: eCAM 2011;2011:342837.

29. Marziali E, McDonald L, Donahue P. The role of coping humor in the physical and mental health of older adults. Aging Ment Health 2008;12:713-8.

30. Bullock M, Russell JA. Preschool children's interpretation of facial expressions of emotion. Int $\mathrm{J}$ Behav Dev 1984;7:193-214.

31. Vagnoli L, Caprilli S, Robiglio A, et al. 
Clown doctors as a treatment for preoperative anxiety in children: a randomized, prospective study. Pediatrics 2005;116:e563-7.

32. Dionigi A, Sangiorgi D, Flangini R. Clown intervention to reduce preoperative anxiety in children and parents: a randomized controlled trial. J Health Psychol 2014;19:369-80.

33. Fernandes SC, Arriaga P. The effects of clown intervention on worries and emotional responses in children undergoing surgery. J Health Psychol 2010;15:40515.

34. Vagnoli L, Caprilli S, Messeri A. Parental presence, clowns or sedative premedication to treat preoperative anxiety in children: what could be the most promising option? Paediatr Anaesth 2010;20:937-43.

35. Bringuier S, Dadure C, Raux O, et al. The perioperative validity of the visual analog anxiety scale in children: a discriminant and useful instrument in routine clinical practice to optimize postoperative pain management. Anesth Analg 2009;109:737-44.

36. Garra G, Singer AJ, Domingo A, et al. The Wong-Baker pain FACES scale measures pain, not fear. Pediatr Emerg Care 2013;29:17-20.

37. von Baeyer CL. Children's self-reports of pain intensity: scale selection, limitations and interpretation. Pain Res Manag 2006;11:157-62.

38. Tomlinson D, von Baeyer CL, Stinson $\mathrm{JN}$, et al. A systematic review of faces scales for the self-report of pain intensity in children. Pediatrics 2010;126:e1168-98.

39. von Baeyer CL, Jaaniste T, Vo HLT, et al. Systematic Review of Self-Report Measures of Pain Intensity in 3- and 4Year-Old Children: Bridging a Period of Rapid Cognitive Development. J Pain 2017;18:1017-26.

40. Bosenberg A, Thomas J, Lopez T, et al. Validation of a six-graded faces scale for evaluation of postoperative pain in children. Paediatr Anaesth
2003;13:708-13.

41. Wolpe J. The practice of behavior therapy: Pergamon; 1973.

42. Tanner BA. Validity of global physical and emotional SUDS. Appl Psychophysiol Biofeedback 2012; 37:31-4

43. Benjamin CL, O’Neil KA, Crawley SA, et al. Patterns and predictors of subjective units of distress in anxious youth. Behav Cogn Psychother 2010;38:497504.

44. Kiyimba N, O'Reilly M. The clinical use of Subjective Units of Distress scales (SUDs) in child mental health assessments: a thematic evaluation. J Mental Health 2017:1-6.

45. Kim D, Bae H, Chon Park Y. Validity of the subjective units of disturbance scale in EMDR. J EMDR Pract Res 2008;2:57-62.

46. Dreger VA, Tremback TF. Management of preoperative anxiety in children. AORN J 2006;84:778-80, 82-6, 88-90 passim; quiz 805-8.

47. Messina M, Molinaro F, Meucci D, et al. Preoperative distraction in children: hand-held videogames vs clown therapy. Pediatr Med Chir 2014;36:98.

48. Tang J, Gibson SJ. A psychophysical evaluation of the relationship between trait anxiety, pain perception, and induced state anxiety. J Pain 2005;6:612-9.

49. Kerimoglu B, Neuman A, Paul J, et al. Anesthesia induction using video glasses as a distraction tool for the management of preoperative anxiety in children. Anesth Analg 2013;117:1373-9.

50. Kim H, Jung SM, Yu H, et al. Video Distraction and Parental Presence for the Management of Preoperative Anxiety and Postoperative Behavioral Disturbance in Children: A Randomized Controlled Trial. Anesth Analg 2015;121:778-84.

51. Rhodes L, Nash C, Moisan A, et al. Does preoperative orientation and education alleviate anxiety in posterior spinal fusion patients? A prospective, randomized study. J Pediatr Orthop 2015;35:276-9.

52. Sadegh Tabrizi J, Seyedhejazi M, Fakhari A, et al. Preoperative Education and Decreasing Preoperative Anxiety Among Children Aged 8-10 Years Old and Their Mothers. Anesthesiol Pain Med 2015;5:e25036.

53. Sola C, Lefauconnier A, Bringuier S, et al. Childhood preoperative anxiolysis: Is sedation and distraction better than either alone? A prospective randomized study. Paediatr Anaesth 2017;27:82734.

54. Yu H, Liu Y, Li S, et al. Effects of music on anxiety and pain in children with cerebral palsy receiving acupuncture: a randomized controlled trial. Int J Nurs Studies 2009;46:1423-30.

55. William Li HC, Lopez V, Lee TL. Effects of preoperative therapeutic play on outcomes of school-age children undergoing day surgery. Res Nurs Health 2007;30:320-32.

56. Dionigi A, Gremigni P. A combined intervention of art therapy and clown visits to reduce preoperative anxiety in children. J Clin Nurs 2017;26:632-40.

57. Agostini F, Monti F, Neri E, et al. Parental anxiety and stress before pediatric anesthesia: a pilot study on the effectiveness of preoperative clown intervention. $\mathrm{J}$ Health Psychol 2014; 19:587-601.

58. Bevan JC, Johnston C, Haig MJ, et al. Preoperative parental anxiety predicts behavioural and emotional responses to induction of anaesthesia in children. Canad J Anaesth 1990;37:177-82.

59. Sanchez JC, Echeverri LF, Londono MJ, et al. Effects of a Humor Therapy Program on Stress Levels in Pediatric Inpatients. Hosp Pediatr 2017;7:46-53.

60. Rimon A, Shalom S, Wolyniez I, et al. Medical Clowns and Cortisol levels in Children Undergoing Venipuncture in the Emergency Department: A Pilot Study. Israel Med Assoc J 2016;18:6803. 